\title{
Barriers to exclusive breast-feeding in Indonesian hospitals: a qualitative study of early infant feeding practices
}

\author{
Valerie J Flaherman ${ }^{1, *}$, Shannon Chan ${ }^{2}$, Riya Desai ${ }^{3}$, Fransisca Handy Agung ${ }^{4}$, \\ Hendri Hartati ${ }^{5}$ and Fitra Yelda ${ }^{5}$ \\ 'Department of Pediatrics, University of California-San Francisco, 3333 California Street, Box 0503, San Francisco, \\ CA 94118 , USA: ${ }^{2}$ School of Medicine, University of California-San Francisco, San Francisco, CA, USA: ${ }^{3}$ University \\ of California-Berkeley, Berkeley, CA, USA: ${ }^{4}$ Faculty of Medicine, University of Pelita Harapan, Banten, Indonesia: \\ ${ }^{5}$ Centre for Health Research, Faculty of Public Health, University of Indonesia, Depok, Indonesia
}

Submitted 30 November 2017: Final revision received 30 April 2018: Accepted 4 May 2018: First published online 5 July 2018

\begin{abstract}
Objective: Although initiating breast-feeding is common in Indonesia, rates of exclusive breast-feeding are low. Our objective was to identify early barriers to exclusive breast-feeding in Indonesian hospitals.

Design: Qualitative. Semi-structured interviews were conducted in April-June 2015. The data were analysed using thematic analysis.

Setting: Indonesian provinces of Jakarta, Banten and West Java.

Subjects: Fifty-four participants including public health officials, hospital administrators, health-care professionals and parents.

Results: Five themes were identified as contributing to low rates of early exclusive breast-feeding in Indonesian hospitals: (i) quality and quantity of breast-feeding education; (ii) marketing and influence of infant formula manufacturers; (iii) hospital infrastructure; (iv) policy, legislation and protocols; and (v) perceived need for infant formula supplementation. Participants noted that providers and mothers receive inadequate or incorrect education regarding breast-feeding; manufacturers promote infant formula use both inside and outside hospitals; constraints in physical space and hospital design interfere with early breastfeeding; legislation and protocols designed to promote breast-feeding are inconsistently enforced and implemented; and providers and mothers often believe infant formula is necessary to promote infant health. All participants identified numerous barriers to early exclusive breast-feeding that related to more than one identified theme.

Conclusions: Our study identified important barriers to early exclusive breastfeeding in Indonesian hospitals, finding that participants consistently reported multifaceted barriers to early exclusive breast-feeding. Future research should examine whether system-level interventions such the Baby-Friendly Hospital Initiative might improve rates of exclusive breast-feeding by improving breastfeeding education, reducing manufacturer influence, modifying existing infrastructure and providing tools needed for protocols and counselling.
\end{abstract}

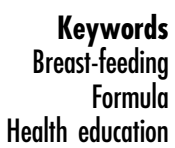

Globally, exclusive breast-feeding for the first 6 months is one of the most effective preventive health measures available to reduce child morbidity and mortality ${ }^{(1-4)}$. Compared with both non-breast-fed infants and mixed-fed infants, exclusively breast-fed infants are significantly less likely to develop diarrhoeal disease, a major cause of infant mortality, and experience a large risk reduction for other diseases as well $^{(1,3,5-7)}$. In the light of these important benefits, the WHO and UNICEF recommend exclusive breast-feeding, without any additional foods or fluids, for the first 6 months with continued breast-feeding for up to 2 years ${ }^{(1,2)}$.
Indonesia is the fourth largest country in the world with a population of 256 million $^{(8)}$. Breast-feeding in Indonesia is almost universal, with $96 \%$ of children under 2 years of age having ever been breast-fed ${ }^{(9,10)}$. However, only $42 \%$ of infants aged $0-5$ months are breast-fed exclusively ${ }^{(9,10)}$. Improving exclusive breast-feeding rates would provide a great public health benefit in Indonesia, especially because one in three households relies on an unsafe water source, increasing an infant's risk of diarrhoea ${ }^{(1,3,5-7,11)}$.

The Indonesian Parliament enacted legislation in 2009 calling for infants to be exclusively breast-fed for the first 
6 months unless medically contraindicated ${ }^{(10,12)}$. The law now states that any person intentionally obstructing breastfeeding may be punished with a monetary fine or with imprisonment for up to 1 year, and that any corporation intentionally obstructing breast-feeding may be subject to a larger fine ${ }^{(10)}$. However, the WHO International Code of Marketing of Breastmilk Substitutes, which bans advertising of breast-milk substitutes and free samples or direct marketing to families or health workers, has not yet been fully adopted by the Indonesian Government ${ }^{(10,13)}$.

Several studies have explored both positive and negative factors associated with breast-feeding initiation and duration in Indonesia. Susiloretni et al. found that mothers with a high level of breast-feeding knowledge had longer duration of exclusive breast-feeding, while lack of familial support and free infant formula samples were associated with shorter exclusive breast-feeding duration ${ }^{(14)}$. Other small studies have reported that exclusive breast-feeding was less common among babies delivered by caesarean section, those with complications during delivery, those delivered in a government or non-health facility and those with unsupportive family members ${ }^{(15-17)}$.

Breast-feeding support that occurs in accordance with best practices such as the Baby-Friendly Hospital Initiative (BFHI) has been shown to increase exclusive breastfeeding rates ${ }^{(18)}$. Although fewer than half of Indonesian births currently occur in a hospital, the proportion has been increasing steadily since $1994^{(19)}$. As of 2011, only $8 \%$ of state public hospitals in Indonesia complied with at least seven of the ten BFHI steps to successful breastfeeding ${ }^{(20)}$. When compared with thirteen other developing countries, Indonesia had the fifth lowest percentage of hospitals to ever be certified as $\mathrm{BFHI}^{(20)}$.

To obtain a comprehensive understanding of barriers to the early establishment of exclusive breast-feeding after delivery in hospital settings, our team conducted and analysed semi-structured interviews from a diverse group of stakeholders including local, district and national public health officials, doctors, midwives and nurses at birthing hospitals, hospital administrators, representatives from professional and community organizations, and mothers of infants recently born in an inpatient setting.

\section{Methods}

We conducted semi-structured interviews with fifty-four participants to explore attitudes, opinions and experiences regarding early breast-feeding support in Indonesian hospitals. We used qualitative methodology to identify themes related to barriers to the establishment of exclusive breast-feeding.

\section{Participants}

Participants were enrolled between January and June 2015 from three different Indonesian provinces: Jakarta, West Java and Banten. To adequately assess the full spectrum of barriers to exclusive breast-feeding during the birth hospitalization, we used typical case purposive sampling to identify potential participants from a wide variety of stakeholders and locations, including public health officials, hospital managers, clinicians, mothers and fathers, representing perspectives from public and private, as well as rural and urban, settings. Mothers and fathers were sampled within the first days postpartum.

Potentially eligible participants received a letter requesting their participation in the study. Those who expressed interest in study participation received a formal letter from the Center for Health Research, University of Indonesia explaining the study and the process of informed consent. If a potential participant continued to express interest in study participation, an interview was scheduled. A short explanation of the study was repeated in-person at the scheduled interview before participants were asked to sign written consent for study participation. Participants who were unable to complete the entire interview were excluded.

The study was conducted according to the guidelines laid down in the Declaration of Helsinki. All procedures involving human subjects were approved by the Research Ethical Committee of the Faculty of Public Health, University of Indonesia and conducted in accordance with the policies of the Institutional Review Board (formerly the Committee on Human Research) of the University of California-San Francisco. Written informed consent was obtained from all subjects.

\section{Data collection}

Data collection methodology was overseen by our study investigator from the Center for Health Research, University of Indonesia (F.H.A.). All interviews were conducted by research assistants who were fluent in Indonesian Bahasa and trained to administer the study's interview guides. The interview guides covered the following topics: (i) current policy and practice regarding hospital support for early breast-feeding; (ii) implementation of recommended hospital breast-feeding practices (including the WHO's Ten Steps to Successful Breastfeeding); and (iii) barriers to promoting early exclusive breast-feeding. Interview guides were tailored to the professional category of the participants (see online supplementary material, Supplemental Tables 1a-g). Interviews were conducted in Indonesian Bahasa, audiorecorded and transcribed, and then translated into English for analysis. All data presented herein were then back-translated into Indonesian by a bilingual investigator (F.H.A.).

\section{Data analysis}

Transcripts were analysed independently for major themes by two of the authors (S.C. and R.D.) using a line-by-line open coding process until thematic categories emerged. After generating an initial coding scheme including a range of observable categories, we then repeated the open coding process using an incident-by-incident coding 
technique and compared these two approaches in the completion of our open coding scheme. During the process of generating an initial coding scheme, coders attempted to identify links between thematic categories, and similarities and differences among thematic categories. All transcripts were coded by both coders, with discrepancies negotiated and resolved with consultation from V.J.F. and F.H.A.

\section{Results}

A total of fifty-four participants (five hospital administrators, eleven doctors, four midwives, six nurses, ten postpartum mothers, ten health department administrators, three representatives from professional organizations and five representatives from community support groups for breastfeeding) were interviewed across three Indonesian provinces: Jakarta (twenty participants from a total of three hospitals), Banten (fourteen participants from a total of two hospitals) and West Java (twenty participants from a total of three hospitals). Participants were $13 \%$ male. Among the five community support group representatives, two were fathers. None of the participants either worked or had given birth in BFHI-certified hospitals. We identified five themes regarding barriers to early exclusive breast-feeding: breastfeeding education; marketing and influence of infant formula manufacturers; infrastructural barriers; hospital policy, protocol and government legislation; and perceived need for infant formula supplementation.

\section{Breast-feeding education (Theme 1)}

Mothers received a spectrum of breast-feeding support from the hospital staff (Table 1). Some mothers reported receiving good breast-feeding support in the hospital:
'When the mothers want to breast-feed, they teach us how to do it.' (Participant 7, mother, Banten, hospital 1)

Other mothers reported a lack of support:

'I think it [education] needs more attention; not every hospital supports breast-feeding for new mothers who have just given birth. Some hospitals resort to infant formula instead because it's more practical.' (Participant 52, mother/representative from community support group, Banten)

Many of the interviewed hospital staff reported a lack of breast-feeding education in their training. Out of the eighteen health professionals who were asked about their breast-feeding training in school, eleven said they received none or very minimal breast-feeding education:

'Oh, I received nothing about breast-feeding.' (Participant 1, nurse, Jakarta, hospital 1)

Staff also reported a lack of on-site training:

'As long as I have worked at the Perinatal Unit, it's been 3 years now, there's no training whatsoever.' (Participant 35, physician, Banten, hospital 2)

'I heard once about the breast milk counselling training, but it's for nurses, not for us.' (Participant 13, midwife, Jakarta, hospital 1)

Many providers also reported never being trained in hand expression of breast milk:

'We have never done it [hand expression] before here.' (Participant 12, physician, Jakarta, hospital 2)

Breast-feeding training was often discussed in general staff meetings, instead of dedicated trainings.

Table 1 Breast-feeding education (Theme 1): representative quotes from semi-structured interviews conducted with public health officials, hospital administrators, health-care professionals and parents $(n 54)$ from eight hospitals in three Indonesian provinces (Jakarta, Banten, and West Java), April-June 2015

'Yes, [breast-feeding education now is] better than [with] my first child which made me not calm because the doctor did not give any information regarding what needs to be done, especially because I was working at that time. The doctor did not give any recommendation about this because it was hard to give exclusive breast milk. My first child was given breast milk for just 3 months and being supplemented with infant formula as well, while now l'm not working and the doctors, hospitals, and nurses are very supportive, especially because my child has to be inpatient.' (Participant 7, mother, Banten, hospital 2)

'But in reality, in the practice, several doctors need to read more deeply about exclusive breast-feeding because we all know that some obstetricians are not familiar with or excited about it. As far I know all paediatricians are enthusiastic. Especially the hospitals in the rural areas, it's difficult to monitor.' (Participant 34, physician/representative of professional organization, Jakarta)

'I was given a book, a guide book with information on ways to breast-feed and foods that must be consumed.' (Participant 9 , mother, West Java, hospital 1)

'Breast-feeding used to be an instant option, even without education. But now, I think this [education] is necessary, even mandatory' (Participant 20, mother, Banten, hospital 1)

'Maybe our human resources is still lacking training and education, the training for midwives must be improved ... Not all of them have received standard training.' (Participant 3 , physician, West Java, hospital 1)

'When we do trainings such as on the Early Breast-feeding Initiative and breast milk, we were asked to do such trainings at least once a year. But since then, we have around ninety-four hospitals, sometimes we just include the Early Breast-feeding Initiative training in a regular meeting.' (Participant 15, health department administrator, Banten)

'No, I have never practised that [hand expression] before ... I don't really understand, and I usually recommend using tools.' (Participant 3, physician, West Java, hospital 1) 


\section{Marketing and the influence of infant formula manufacturers (Theme 2)}

Participants observed that hospitals have tried to limit infant formula use after the implementation of breastfeeding laws (Table 2):

'Because after knowing there is such a law, I stopped accepting any offers from the infant formula salesforce.' (Participant 13, midwife, Jakarta, hospital 1)

Some hospitals also reported not advertising brand names of infant formula:

'Even though we have infant formula here, the infant formula is unlabelled, so the boxes are tossed away, then we place the milk in a place without a label. So, we just write [regular] infant formula, infant formula for baby with allergy, infant formula for low birth weight baby, so the brand is not there.' (Participant 1, nurse, Jakarta, hospital 1)

Most hospital administrators stated that they did not have any partnership with infant formula companies:

'As long as I have been here, none. We never ask for sponsorships.' (Participant 40, hospital administrator, West Java, hospital 1)

However, some participants described that infant formula companies continue to have an influence on breastfeeding in and out of the hospital:

'I see many midwives in clinic that work together with the milk company.' (Participant 14, nurse, Banten, hospital 1)
A midwife reported that her recent breast-feeding training was received from a seminar organized by an infant formula manufacturer.

Many of the participants also reported a lack of control over hospital infant formula. Staff members directly interacting with mothers and infants said they had no part in hospital policy making, because infant formula was purchased and distributed by the other departments such as nutrition or pharmacy:

'Maybe they come to the pharmacy, not to us.' (Participant 28, nurse, West Java, hospital 3)

'Infant formula is accepted by the nutrition section ... so if the babies who have an indication to be given infant formula by the doctor, the infant formula must be retrieved from the nutrition department.' (Participant 13, midwife, Jakarta, hospital 1)

Similarly, parents felt little control over whether their infants were fed infant formula while in the hospital:

'Maybe to stop it from crying when I was not yet able to breast-feed, my baby was probably given infant formula ... I don't know.' (Participant 10, mother, Jakarta, hospital 1)

A father reported that:

'If it's not rooming-in, we will never know whether the baby is given infant formula or not.' (Participant 4, representative of community support group, West Java)

Table 2 Marketing and the influence of infant formula companies (Theme 2): representative quotes from semi-structured interviews conducted with public health officials, hospital administrators, health-care professionals and parents $(n 54)$ from eight hospitals in Indonesian provinces (Jakarta, Banten, and West Java), April-June 2015

'When it's not rooming-in, my child was given infant formula. My wife got a goodie bag from Nutricia when she was discharged from the hospital. The next day, Nutricia called me, asking about how my child was doing. I asked her/him, "Who did you get my number from? I never contacted anyone from infant formula company." She/he answered, "Perhaps from our medical colleague." "Why did you call to ask how my child was doing? Even my child's doctor never calls to ask about it," I told her/him. I asked her/him repeatedly from whom they got my number from until finally they turned off the phone.' (Participant 4, father/representative of community support group, West Java)

'[Personally, did you ever get an offer from the infant formula manufacturer?] Yes, but I rejected it, there was one willing to give me percentage, but still I did not want it.' (Participant 14, nurse, Banten, hospital 1)

'[Did any infant formula manufacturer approach this hospital?] There was one [in the past]. But because now we are from Indonesian Midwifery Association and there are a lot of educators, not anymore now. They don't dare.' (Participant 13, midwife, Jakarta, hospital 1)

'In the past it was very popular among midwives to include infant formula in their [service] packages, now we can't do that.' (Participant 26 , midwife/representative of professional organization, West Java)

'I can say that the pressure [from formula manufacturers] still exists, but is not that bad. However, in the hospital and private obstetrics, I can see that they are changing. They no longer use infant formula. Package from RSIA [Maternal Child Hospital] would only contain baby soap. They used to give infant formula in such package, but now they only give baby soap' (Participant 15 , health department administrator, Banten)

'Information from the health official is also clear regarding the problems with [marketing of] infant formula, [but] sometimes people still consider infant formula is better than breast milk, that's the problem. Currently it's getting better, no longer [having] television broadcast means no longer [having] advertisements. But still they are promoting to hospitals, and to nurses, continuously giving samples, and even free samples.' (Participant 3, physician, West Java, hospital 1)

'The health people are the manufacturer's agents ... Yes, it seems that they promote their products through the health programme route.' (Participant 8, health department administrator, Banten)

'To be honest, we never know what the nurses do to babies in the nursery. I don't want to accuse, but many nurses give infant formula to newborns.' (Participant 4, representative of community support group, West Java) 
Infrastructural barriers (Theme 3)

Participants of all types reported infrastructural barriers that prevented successful breast-feeding (Table 3):

'A common reason [for using formula] is that [mothers] are shy to breast-feed in public [wards].' (Participant 12, physician, Jakarta, hospital 2)

'In terms of rooming-in, we are limited by space as there are no specially allocated rooms for it.' (Participant 40, hospital administrator, West Java, hospital 1)

However, many mothers and staff expressed positive sentiments about rooming-in and the effect on breastfeeding:

'So if the baby is born normally, we tend to treat them in the same room to strengthen the bond between mother and baby.' (Participant 12, physician, Jakarta, hospital 2)

'If the condition of the mother and baby allows it, then we do rooming-in. It has so many advantages, first the mother will become accustomed to taking care of the child, so she can be guided how to hold and breast-feed the baby. We can do direct education.' (Participant 39, physician, West Java, hospital 1)

Mothers with infants in the neonatal intensive care unit reported a particularly hard time with infrastructure. Only two surveyed hospitals reported having a dedicated refrigerator to store breast milk if mothers had been discharged. Rapid turnover of trained staff was also a frequently reported obstacle to successful breast-feeding education and promotion:

'That's the problem, [staff] rotation and turnover are very fast. Sometimes [staff rotation] occurs two times, and a minimum of once a year.' (Participant 8 , health department administrator, Banten).

Further, a reported lack of lactation specialists contributed to a further understaffed and under-resourced health care environment:

'We don't have a special staff to handle lactation, so that the nurses on the Children's Unit do double jobs.' (Participant 46, physician, Jakarta, hospital 2)

\section{Hospital protocol and policy/government legislation (Theme 4)}

Most of the staff members had general knowledge of government legislation regarding breast-feeding but cannot recall specifics (Table 4):

'I probably heard of [the breast-feeding law], but not too much into, so maybe I heard, but I forgot.' (Participant 50, physician, Banten, hospital 1)

In terms of hospital policy regarding infant formula supplementation, some hospital staff members reported an informed consent policy whereby mothers who choose to supplement with infant formula are required to sign a consent form:

'If we have to give infant formula to a baby, we'll ask for the family's consent, we'll also need the family to fill in a form.' (Participant 36, nurse, Banten, hospital 2)

Local governments/provinces reported some problems with breast-feeding implementation:

'But there is still no punishment if the hospital doesn't do exclusive breast-feeding, then there is still infant formula promotion, something like that. No punishment yet, or warning, still no.' (Participant 29, health department administrator, West Java)

Table 3 Infrastructural barriers to early exclusive breast-feeding (Theme 3): representative quotes from semi-structured interviews conducted with public health officials, hospital administrators, health-care professionals and parents ( $n$ 54) from eight hospitals in three Indonesian provinces (Jakarta, Banten, and West Java), April-June 2015

'For instance, when the room is quite far away, while the baby's room is here and the NICU room is there, it is quite far. And one more thing, it should be designed properly. A hospital must have a lactating corner, one is enough, but is it really enough? The NICU room doesn't have it, we can't say that every place where there are babies must have a lactating corner, the truth is it doesn't.' (Participant 18, hospital administrator, Jakarta, hospital 1)

'Yes, so from one side, the mother has to breast-feed the baby, but on the other hand, we don't have enough space [for the baby's crib]. There was one time where we put patients in the hallway.' (Participant 13, midwife, Jakarta, hospital 1)

'The parents still need to breast-feed but at the same time there is no privacy ... they don't humanize the mothers who are breast-feeding, because many times they need to open their breasts in front of [other] fathers. So, the privacy is very low.' (Participant 5, physician, Jakarta, hospital 1)

'[We need] a special room for mothers whose babies are still under treatment. Sometimes we get confused, the baby is still being treated, but the mother has been allowed to go home. Sometimes there are mothers who don't want to go home, but if she doesn't want to go home, we don't have enough space and they don't want to move to a higher [more expensive] class.' (Participant 13, midwife, Jakarta, hospital 1)

'As per our evaluation yesterday, overall we are lacking nurses and midwives, and we are in the process of recruiting more, especially for perinatology and the delivery rooms, we need twelve midwife-nurses.' (Participant 40, hospital administrator, West Java, hospital 1)

'Because the number of babies here are quite a lot with a few nurses, sometimes we are forced to replace the breast milk with infant formula when the waiting time is too long:' (Participant 5, physician, Jakarta, hospital 1)

'Yes, it was difficult to see my [first] baby, I could only see her through the glass window. The room was separated. Now, it has been easier because of this "rooming-in" scheme. They should have done that a long time ago.' (Participant 20, mother, West Java, hospital 2)

'I think this kind of treatment [rooming-in] is better than the separation. If the baby gets separated, it would be more hassle; on the other hand, it's easier to control and also breast-feed my baby.' (Participant 2, mother, Jakarta, hospital 2)

NICU, neonatal intensive care unit. 
Table 4 Hospital protocol and policy/government legislation (Theme 4): representative quotes from semi-structured interviews conducted with public health officials, hospital administrators, health-care professionals and parents $(n 54)$ from eight hospitals in three Indonesian provinces (Jakarta, Banten, and West Java), April-June 2015

'We haven't fully disseminated [the law]. But when we do, we would disseminate it to schools, such as the midwifery academy, the medical faculty and the nursing academy.' (Participant 15, health department administrator, Banten)

'But we can't do it right away because we are working with the government, we have to wait for the budgeting at the end of the year, this happens when we're dealing with government money, not private money.' (Participant 6, health department administrator, Banten)

'The problem is we must have a written policy for hospitals. It needs the cooperation of doctors, quality control and the management so it can be circulated fully and more effectively. Also, the Ten Steps to Successful Breastfeeding, trainees should be trained well, it cannot be just read and straightaway applied. When I look at the results of the previous Indonesia Demography [and] Health Survey report in 2012, the hospital still hasn't played its role, it is still not optimal.' (Participant 33, nutritionist/representative of professional organization, West Java)

'Sometimes we meet with the hospital director, but they do not forward the meeting to their subordinates. If we invite the subordinates to explain it to their supervisors, it doesn't reach to [them]. So, it's difficult, we invite the supervisor so they can decide regulation, but they don't come.' (Participant 29, health department administrator, West Java)

'What punishment? Nowadays it's difficult to apply the punishment because it's not reaching that yet. There are other people who violated other regulations without punishment, let alone giving breast-milk substitute to the children. I am so confused.' (Participant 39 , physician, West Java, hospital 1)

'The obstacle is ... the promotion. The rapid promotion of infant formula, many BPM [community development agency] who still give infant formula to the babies. We cannot trace them. Hospitals also do the same thing; giving infant formula to babies, and so do the doctors.' (Participant 49, health department administrator, Jakarta)

'We have the informed consent. If the patient feels that the breast-milk supply is still low, usually she starts to bottle-feed on the second day. Actually, it's no problem even if the patient has gone through C-section, no matter the reason is. So, the mother must give her breast milk. If she insists although we have educated her, she can fill in the informed consent.' (Participant 54, midwife, Jakarta, hospital 3 )

'I know that in [name of hospital], they have a lactation or breast-feeding programme that ensure mothers to breast-feed their children ... but if they insist on giving infant formula, the mother has to sign a written statement confessing her own will to give one, not from the hospital ... they need to make a written statement if they want to give infant formula instead.' (Participant 6, health department administrator, Banten)

'Sorry, I didn't memorize [the law] off the top of my head, but the more important thing is that I know about it and I know how to look it up. This information is in my document. I know that if we try to deter someone from breast-feeding, there can be a punishment for it.' (Participant 1, nurse, Jakarta, hospital 1)

'I've heard it [the breast-feeding law] before but I have never read it.' (Participant 3, physician, West Java, hospital 1)

'It must be only breast milk, not water, and they get scared "Ah ... my baby will get hungry." Yeah, that's what we have to teach, hard challenge to reassure the mother. But patients mostly give up. That's why we have a consent form if she wants to cancel, in order to avoid being blamed, because it's not us who suggest it, but the patients ask for it.' (Participant 22, nurse, West Java, hospital 2)

'The problem [with enforcing legislation] is located in its practice, it turns out that we don't have the legal force. I meant not that we don't have the legal force, but we are not consistent ourselves. It is intended that people who don't breast-feed will be punished, but we haven't practised it yet.' (Participant 8 , health department administrator, Banten)

'We will see the law first; the health law says that there will be a consequence if it's violated, but we don't have any local regulations yet. We can actually make a law, but we have to coordinate with the health service about whether or not they want to give us permission, but there has not been any law implemented about not doing the breast-feeding.' (Participant 6, health department administrator, Banten)

Table 5 Perceived need for infant formula supplementation (Theme 5): representative quotes from semi-structured interviews conducted with public health officials, hospital administrators, health-care professionals and parents $(n 54)$ from eight hospitals in three Indonesian provinces (Jakarta, Banten, and West Java), April-June 2015

'In the past we got carried away by the Western culture that is misleading ... infant formula actually comes from overseas not local product. In the past, we used breast milk actually.' (Participant 8 , health department administrator, Banten)

'There also perspective that giving infant formula would make the baby fatter. This is the case for some here in Serang because the level of education here is not that high.' (Participant 14, nurse, Banten, hospital 1)

'I think the only difficulty that we are facing is that there is no lactation counsellor, so when there is a mother, especially a mother who just has had her first child and having difficulty in breast-feeding, the mother tends to use infant formula even though breast-feeding is still a way that can be pursued.' (Participant 6 , health department administrator, Banten)

'Panic from the family also plays a role, I once worked on the second floor and there was a panic from a family, sometimes the baby cried all the time. So because the baby kept on crying, I bought the infant formula because the mother wasn't convinced that her breast milk would comfort the baby.' (Participant 13, midwife, Jakarta, hospital 1)

'They said I could breast-feed the baby. Even though the milk hasn't come out, they told me to keep trying.' (Participant 21, mother, Banten, hospital 1)

\section{Perceived need for infant formula supplementation (Theme 5)}

Many mothers and their families believed that breast milk is the healthiest option but were still comfortable turning to infant formula as a healthy alternative (Table 5):

'They usually argue that their first child was fine getting infant formula. Even though we explain that breast milk has other nutrients that are irreplaceable by infant formula.' (Participant 1, nurse, Jakarta, hospital 1)

Mothers often reported concern over milk supply:

'Breast milk is better than infant formula. But my problem has been about the breast milk is not enough.' (Participant 2, mother, Jakarta, hospital 2) 
Mothers reported mixed responses from staff; some staff did not know how to correctly support mothers prior to the onset of copious milk production and would simply encourage mothers to keep trying:

'According to the nurses, we just have to keep breast-feeding, it will eventually come out.' (Participant 47, mother, Jakarta, hospital 1)

Other mothers remembered staff being quick to provide infant formula.

Working mothers faced an especially difficult barrier to exclusive breast-feeding:

'I don't have any idea [of breast-feeding support groups] because I am working. I rarely make contact with my neighbours.' (Participant 9, mother, West Java, hospital 1)

'Maybe we would need it [infant formula] once or twice, for example when I'm out for work, because I don't have time, while the baby needs to be fed all the time at home.' (Participant 7, mother, Banten, hospital 1)

Some women felt breast-feeding at work was inappropriate:

'I have a colleague in my office, I'm not saying that she's wrong, but just improper. She pumped all the time and neglected her job. Friends were gossiping about her.' (Participant 20, mother, West Java, hospital 2)

\section{Discussion}

In our study, participants reported a wide variety of barriers to exclusive breast-feeding including lack of breastfeeding education, infant formula marketing, hospital infrastructure, inadequate government and hospital policy, and a perceived need for infant formula supplementation. Participants reported both barriers that might be changed expeditiously and barriers for which change might be more difficult. For example, lack of clarity in hospital protocols for breast-feeding might be relatively straightforward to correct by revising such protocols. However, other barriers, such as lack of space for mothers and babies to room-in together, may be more costly or difficult to modify. Barriers such as maternal and provider perception of the need for infant formula supplementation have been previously reported to be difficult to overcome, but might be amenable to change with increased education and awareness. The BFHI has been shown to be effective at improving exclusive breast-feeding rates in settings where rates of exclusive breast-feeding have previously been low ${ }^{(18,21,22)}$. A comprehensive programme such as the BFHI may potentially provide great benefit because of its demonstrated ability to address multiple barriers to breast-feeding simultaneously, such as improving knowledge of health-care providers, improving hospital policy and improving maternal education ${ }^{(23)}$.

Although our study identified many barriers to exclusive breast-feeding during the birth hospitalization, many facilitators were identified as well. Consistent with the high rates of initiation of at least some breast-feeding in Indonesia, breast-feeding (although perhaps not exclusive breast-feeding) was considered a normal part of the birth experience. Overall, participants perceived that a breastfeeding mother may need support and education, even while they may have been unsure how to deliver that support. Participants also had a general awareness of the federal legislation protecting breast-feeding and acknowledged its importance.

However, despite participants having a general awareness of existing policy and legislation in Indonesia, compliance with existing policies and legislation in support of exclusive breast-feeding was reported to be inconsistent, and participants did not describe actual enforcement of existing legislation. Inconsistent enforcement of breastfeeding-related legislation has been widely reported in other countries and may contribute substantially to the low rates of exclusive breast-feeding in Indonesia ${ }^{(24,25)}$. Further, our study corroborates previous findings about the continuing impact of infant formula marketing on health workers and family decision making in regard to breastfeeding ${ }^{(26)}$. Marketing of breast-milk substitutes targets mothers and health workers which undermines and disincentivizes breast-feeding, thus increasing costs at the household level ${ }^{(13)}$. To improve exclusive breast-feeding rates in Indonesia, the government should fully adopt and implement the WHO code banning advertising of breastmilk substitutes ${ }^{(27,28)}$. Stricter policy action from the government is required to suppress the encroachment of infant formula companies in health-care settings and their influence through community marketing and advertising.

Lack of accessibility to staff members with expertise in breast-feeding was cited by many participants as a major barrier to promotion of exclusive breast-feeding. Participants reported that specialist support from lactation consultants was lacking, while at the same time general medical staff such as physicians, nurses and midwives had not received training in breast-feeding support. Improving accessibility to staff members with expertise in breastfeeding might also help address other obstacles to exclusive breast-feeding such as inadequate maternal breast-feeding education and maternal perception of insufficient milk supply. Especially for first-time mothers, staff breast-feeding expertise may be essential in supporting optimal breastfeeding and promoting exclusivity because infant formula was often used when breast-feeding support was lacking. Our findings concur in this regard with previous studies in this area, which have shown that breast-feeding support provided by trained clinicians significantly improves maternal self-efficacy, duration of exclusive breast-feeding and confidence of staff when counselling mothers ${ }^{(29-31)}$. 
Support from family members can also improve breastfeeding self-efficacy and duration of breast-feeding ${ }^{(27,28)}$, but our study participants did not describe many experiences related to family support. Further research is needed in this crucial area.

Our study has several important limitations. First, our participants were located in three Indonesian provinces. Since Indonesia is a large, socio-economically and culturally diverse country, further research is needed to determine if the factors identified in these three provinces are applicable in the other thirty-one provinces not studied. Second, all participants in our study consented to participate. It is possible that doctors, nurses and mothers who participated in these interviews might have a greater interest in and commitment to breast-feeding than those who did not participate. Perceived barriers to exclusive breast-feeding might be different for doctors, mothers and nurses who did not choose to participate. Third, our typical case sampling methodology may not have captured the full variation of barriers to exclusive breastfeeding and our sample size was too limited to allow us to compare barriers by location. In addition, we did not collect demographic information on participants. Additional sampling methods with larger sample sizes and demographic data collection may be necessary to obtain a more complete understanding of barriers and how these may vary between hospitals and provinces.

\section{Conclusion}

Our study identified multiple factors that may present barriers to exclusive breast-feeding in Indonesia, including infant formula marketing, hospital protocol, legislation, infrastructure problems, lack of breast-feeding education and perceived need for infant formula supplementation. Interventions such as the BFHI may help address these barriers by providing improved breast-feeding education, developing breast-feeding policies and addressing maternal and provider concerns about the perceived need for infant formula. Further research is needed to examine whether implementation of the BFHI or other systemlevel interventions, including improved clinical guidelines, can increase rates of exclusive breast-feeding in Indonesia.

\section{Acknowledgements}

Acknowledgments: The authors would gratefully like to thank Dr Sabarinah Prasetyo for her contribution to the conception of the study and its methodology, Dr Utami Roesli who conceptualized the study, Ms Hikmah Kurniasari for her help with developing the research infrastructure and Dr Wiyarni Pambudi who assisted with recruitment and data collection. Financial support: This work was supported by the US Agency for International
Development (USAID) (grant number AID-OAA-A-1100012). USAID had no role in the design, analysis or writing of this article. Conflicts of interest: The authors declare no conflict of interest. Authorship: V.J.F. participated in study design and data analysis and revised the manuscript for important scientific content. S.C. and R.D. participated in data analysis and drafted the manuscript. F.H.A. conceived and designed this study, participated in data analysis and revised the manuscript for important scientific content. H.H. and F.Y. participated in data collection and analysis and revised the manuscript for important scientific content. Ethics of buman subject participation: This study was conducted according to the guidelines laid down in the Declaration of Helsinki. All procedures involving human subjects were approved by the Research Ethical Committee of the Faculty of Public Health, University of Indonesia and conducted in accordance with the policies of the Institutional Review Board (formerly the Committee on Human Research) of the University of California-San Francisco. Written informed consent was obtained from all subjects.

\section{Supplementary material}

To view supplementary material for this article, please visit https://doi.org/10.1017/S1368980018001453

\section{References}

1. UNICEF \& World Health Organization (2009) Baby-Friendly Hospital Initiative: Revised, Updated and Expanded for Integrated Care. Section 1, Background and Implementation. http://apps.who.int/iris/bitstream/handle/10665/43593/978 9241594967_eng.pdf;jsessionid=A7048A190A163CD682499 D1714AF7746? sequence $=1$ (accessed May 2018).

2. Saadeh RJ (2012) The Baby-Friendly Hospital Initiative 20 years on: facts, progress, and the way forward. J Hum Lact 28, 272-275.

3. UNICEF (2015) Nutrition: Breastfeeding, Risks of Artificial Feeding. http://www.unicef.org/nutrition/index_24824.html (accessed July 2015).

4. Perez-Escamilla R, Martinez JL \& Segura-Perez S (2016) Impact of the baby-friendly hospital initiative on breastfeeding and child health outcomes: a systematic review. Matern Child Health J 12, 402-417.

5. Sankar MJ, Sinha B, Chowdury R et al. (2015) Optimal breastfeeding practices and infant and child mortality: a systematic review and meta-analysis. Acta Paediatr 104, 3-13.

6. Bowatte G, Tham R, Allen KJ et al. (2015) Breastfeeding and childhood acute otitis media: a systematic review and metaanalysis. Acta Paediatr 104, 85-95.

7. Haroon S, Jas JK, Salam RA et al. (2013) Breastfeeding promotion interventions and breastfeeding practices: a systematic review. BMC Public Health 13, Suppl. 3, S20.

8. Central Intelligence Agency (2015) The World Factbook. https:/www.cia.gov/library/publications/the-world-factbook/ rankorder/2119rank.html (accessed July 2015).

9. Statistics Indonesia, National Population and Family Planning Board, Ministry of Health, et al. (2013) Indonesia Demographic and Health Survey 2012. Jakarta: BPS, BKKBN, Kemenkes and ICF International. 
10. Soekarjo A \& Zehner E (2011) Legislation should support optimal breastfeeding practices and access to low-cost, high-quality complementary foods: Indonesia provides a case study. Matern Child Nutr 7, Suppl. 3, 112-122.

11. UNICEF Indonesia (2012) Water, sanitation \& hygiene. Issue Briefs, October 2012. https://www.unicef.org/indonesia/ A8-_E_Issue_Brief_Water_Sanitation_REV.pdf (accessed May 2018).

12. Shetty P (2014) Indonesia's breastfeeding challenge is echoed the world over. Bull World Health Organ 92, 234-235.

13. Save the Children (2013) Superfood for Babies: How Overcoming Barriers to Breastfeeding will Save Children's Lives. https://www.savethechildren.org.uk/content/dam/ global/reports/health-and-nutrition/superfood-for-babiesUK-version.pdf (accessed May 2018).

14. Susiloretni KA, Hadi H, Prabandari YS et al. (2015) What works to improve duration of exclusive breastfeeding: lessons from the exclusive breastfeeding promotion program in rural Indonesia. Matern Child Health J 19, 1515-1525.

15. Titaley CR, Loh PC, Prasetyo S et al. (2014) Socio-economic factors and use of maternal health services are associated with delayed initiation and non-exclusive breastfeeding in Indonesia: secondary analysis of Indonesia Demographic and Health Surveys 2002/2003 and 2007. Asia Pac J Clin Nutr 23, 91-104.

16. Februhartanty J, Wibowo Y, Fahmida U et al. (2012) Profiles of eight working mothers who practiced exclusive breastfeeding in Depok, Indonesia. Breastfeed Med 7, 54-59.

17. Basrowi RW, Sulistomo AB, Adi NP et al. (2015) Benefits of a dedicated breastfeeding facility and support program for exclusive breastfeeding among workers in Indonesia. Pediatr Gastroenterol Hepatol Nutr 18, 94-99.

18. Howe-Heyman A \& Lutenbacher M (2016) The BabyFriendly Hospital Initiative as an intervention to improve breastfeeding rates: a review of the literature. J Midwifery Womens Health 61, 77-102.

19. Titaley CR, Dibley MJ \& Roberts CL (2012) Type of delivery attendant, place of delivery and risk of early neonatal mortality: analyses of the 1994-2007 Indonesia Demographic and Health Surveys. Health Policy Plan 27, 405-416.
20. Labbok MH (2012) Global baby-friendly hospital initiative monitoring data: update and discussion. Breastfeed Med 7, 210-222.

21. Braun ML, Giugliani ER, Soares ME et al. (2003) Evaluation of the impact of the baby-friendly hospital initiative on rates of breastfeeding. Am J Public Health 93, 1277-1279.

22. Kramer MS, Guo T, Platt RW et al. (2003) Infant growth and health outcomes associated with 3 compared with 6 mo of exclusive breastfeeding. Am J Clin Nutr 78, 291-295.

23. VanDevanter N, Gennaro S, Budin W et al. (2014) Evaluating implementation of a baby friendly hospital initiative. MCN Am J Matern Child Nurs 39, 231-237.

24. Berry NJ \& Gribble KD (2017) Health and nutrition content claims on websites advertising infant formula available in Australia: a content analysis. Matern Child Nutr 13, e12383.

25. Rollins NC, Bhandari N, Hajeebhoy N et al. (2016) Why invest, and what it will take to improve breastfeeding practices? Lancet 387, 491-504.

26. Hidayana I, Februhartanty J \& Parady VA (2017) Violations of the international code of marketing of breast-milk substitutes: Indonesia context. Public Health Nutr 20, 165-173.

27. Odom EC, Li R, Scanlon KS et al. (2014) Association of family and health care provider opinion on infant feeding with mother's breastfeeding decision. J Acad Nutr Diet 114, 1203-1207.

28. Rempel LA, Rempel JK \& KCJ Moore (2017) Relationships between types of father breastfeeding support and breastfeeding outcomes. Matern Child Nutr 13, e12237.

29. Imdad A, Yakoob MY \& Bhutta ZA (2011) Effect of breastfeeding promotion interventions on breastfeeding rates, with special focus on developing countries. BMC Public Health 11, Suppl. 3, S24.

30. Carvalho de Jesus P, Couto de Oliveira MI \& Fonseca SC (2016) Impact of health professional training in breastfeeding on their knowledge, skills, and hospital practices: a systematic review. J Pediatr (Rio J) 92, 436-450.

31. Labarere J, Gelbert-Baudino N, Ayral AS et al. (2005) Efficacy of breastfeeding support provided by trained clinicians during an early, routine, preventive visit: a prospective, randomized, open trial of 226 mother-infant pairs. Pediatrics 115, e139-e146. 